\title{
Trastornos metabólicos renales en pacientes urolitiásicos cubanos mayores de 60 años
}

\author{
Raymed A. Bacallao-Méndez ${ }^{1 *}$, Reynaldo Mañalich-Comas², Francisco Gutiérrez-García1, \\ Inés M. Roig-Duarte ${ }^{2}$, Médelin R. Reyes-González ${ }^{1}$ y Víctor J. Medranda-Salgado ${ }^{1}$
}

${ }^{1}$ Departamento de Docencia e Investigaciones; 'Departamento de Fisiopatología Renal. Instituto de Nefrología "Dr. Abelardo Buch López". La Habana, Cuba

\section{Resumen}

Objetivos: Identificar los trastornos metabólicos renales de la población litiásica cubana de 60 años y mayor, y la relación de los más frecuentes con variables demográficas y antropométricas. Material y métodos: Estudio descriptivo y transversal de pacientes cubanos de 60 años de edad y mayores con litiasis urinaria, sometidos a estudio metabólico renal en el Instituto de Nefrología, La Habana, Cuba, en el período de 2010 a 2019. La información se procesó de forma automatizada (SPSS 22.0). Se utilizó análisis de distribución de frecuencias, promedios y la prueba de independencia. Resultados: Los trastornos metabólicos más comunes identificados fueron hipomagnesuria (43.9\%), excreción de sodio aumentada (39.9\%) y pH urinario ácido (32.4\%). La hiperuricemia experimentó el mayor incremento con el aumento de la edad ( $p<0.05)$. La mayor excreción de sodio y la hipercalciuria fueron significativamente más frecuentes en pacientes desnutridos y con pesos normales $(51.2 \%$ y $15.4 \%)$ ( $p$ <.01). Conclusiones: Los trastornos metabólicos renales más comunes en pacientes litiásicos cubanos mayores de 60 años son hipomagnesuria, excreción urinaria de sodio aumentada y pH urinario ácido. La hiperuricemia es más frecuente en pacientes de más de 74 años. La excreción urinaria de sodio incrementada y la hipercalciuria son más comunes en pacientes desnutridos y de peso normal.

Palabras clave: Trastornos metabólicos renales. Urolitiasis. Cálculos urinarios. Adulto mayor. Hipomagnesuria. Hiperuricemia.

\section{Renal metabolic disturbances in Cuban urolithiasic patients over 60 years}

\section{Abstract}

Objectives: To identify the renal metabolic disorders present in the Cuban lithiasic population aged 60 years and over, and the relationship of those more frequent with demographic and anthropometric variables. Materials and methods: Descriptive, cross-sectional study of Cuban patients aged 60 and over with urinary lithiasis, who underwent a renal metabolic study at the Institute of Nephrology, Havana, Cuba, in the period 2010-2019. The information was processed in an automated way (SPSS22.0). The analysis of frequency distribution, means and the independence test were used. Results: The most common metabolic disorders found were hypomagnesuria (43.9\%), increased sodium excretion (39.9\%) and acidic urinary $\mathrm{pH}(32.4 \%)$. Hyperuricemia experienced the greatest increase with increasing age ( $p<0.05)$. Increased sodium excretion and hypercalciuria were significantly more frequent in malnourished and normal weight patients $(51.2 \%$ and $15.4 \%)(p<0.01)$.

\section{Correspondencia:}

*Raymed A. Bacallao-Méndez E-mail: raymed@infomed.sld.cu
Fecha de recepción: 09-10-2020

Fecha de aceptación: 03-01-2021

DOI: 10.24875/NEFRO.20000051
Disponible en internet: 18-06-2021

Nefro Latinoam. 2021;18:33-40 www.nefrologialatinoamericana.com

2444-9032/@ 2021 Sociedad Latinoamericana de Nefrología e Hipertensión. Publicado por Permanyer. Este es un artículo open access bajo la licencia CC BY-NC-ND (http://creativecommons.org/licenses/by-nc-nd/4.0/). 
Nefro Latinoam. 2021;18

Conclusions: The most common renal metabolic disorders in Cuban lithiasic patients older than 60 years are hypomagnesuria, increased urinary sodium excretion, and acid urinary $\mathrm{pH}$. Hyperuricemia is more common in patients over 74 years of age. Increased urinary sodium excretion and hypercalciuria are more common in malnourished and normal weight patients.

Key words: Renal metabolic disorders. Urolithiasis. Urinary calculi. Aged. Hypomagnesuria. Hyperuricemia.

\section{Introducción}

La litiasis urinaria es una de las enfermedades de las que se tiene conocimiento desde la antigüedad; sin embargo, pese al conocimiento acumulado, su prevalencia se incrementa en el mundo'. La urolitiasis se caracteriza por su elevada recurrencia y alto efecto económico ${ }^{2,3}$. En Cuba, la enfermedad renal crónica de origen obstructivo (en su mayoría, urolitiasis) constituye la quinta causa de indicación de métodos sustitutivos de la función renal con el $4.5 \%$ en $2014^{4}$.

Otro fenómeno actual es el envejecimiento poblacional, según la Organización Mundial de la Salud, que define la vejez como vivir más de 60 años en los países en desarrollo y 65 en los desarrollados, en 2025 vivirán más de 1200 millones de personas mayores de 60 años en el mundo ${ }^{5,6}$. En Cuba se calcula que en el año 2030 el $30.3 \%$ de la población tendrá más de 60 años de edad'. Esto se acompaña de un incremento del número de sujetos considerados obesos y con sobrepeso, que según la última encuesta nacional de factores de riesgo constituyen el $43.8 \%$ de la población adulta ${ }^{8}$.

La obesidad y los estados de resistencia a la insulina se suelen acompañar en general de modificaciones metabólicas que propician la litogénesis ${ }^{9}$. Sin embargo, los trastornos metabólicos renales que producen la litogénesis se han estudiado poco en pacientes ancianos a escala global y en Latinoamérica ${ }^{10}$, lo que limita el desarrollo de medidas encaminadas a prevenir las urolitiasis y sus recurrencias en este grupo poblacional, de tal modo que este estudio se desarrolla con el objetivo de identificar los trastornos metabólicos urinarios de la población litiásica cubana de 60 años y mayor, y la relación de los más frecuentes con variables demográficas y antropométricas.

\section{Material y métodos}

Se realizó un estudio observacional descriptivo de corte transversal. El universo de estudio estuvo constituido por todos los pacientes cubanos de 60 años de edad y mayores, con litiasis urinaria (demostrada por estudios imagenológicos, o eliminación espontánea o instrumental), procedentes de todo el país, que acudieron a realizarse estudio metabólico renal al Instituto de Nefrología "Dr. Abelardo Buch López" (centro de atención terciaria), en La Habana, Cuba, en el período comprendido entre enero del año 2010 y diciembre del 2019.

Sólo se incluyó el primer estudio metabólico realizado a cada individuo. Se excluyó a los individuos con muestras mal recolectadas, los pacientes extranjeros, los vegetarianos, aquéllos con incontinencia urinaria o trastornos en el vaciamiento vesical, así como individuos con distrofias musculares, amputados y con función renal por depuración de creatinina < $30 \mathrm{ml} /$ $\mathrm{min} / 1.73 \mathrm{~m}^{2} \mathrm{SC}$. Además, no se consideró a los pacientes que habían presentado un cólico renal en las últimas seis semanas, tuvieran tratamiento de reemplazo hormonal o los sometidos a una operación bariátrica previa.

La información se recogió a partir de los informes de resultados de los estudios metabólicos efectuados en el período, de los que se tomaron los datos correspondientes a las variables siguientes: edad, sexo, peso, talla, volumen urinario, concentraciones plasmáticas de creatinina, calcio, fósforo, ácido úrico y excreción urinaria en 24 horas de creatinina, sodio, calcio, fósforo, ácido úrico, magnesio, oxalato y citrato. Asimismo, se registraron el $\mathrm{pH}$ urinario y la presencia o no de cistinuria e infección del tracto urinario (ITU).

Todos los sujetos fueron objeto de un breve interrogatorio para asegurar que no cumplieran ningún criterio de exclusión; se les suspendió toda la medicación cinco días antes del estudio (salvo que implicara un riesgo vital y siempre que no incluyera diuréticos, alcalinizantes urinarios o hipouricémicos) y se les recogió una muestra de orina de $24 \mathrm{~h}$ (en frascos lavados y con timol al 5\% como conservador) con su dieta habitual (ad libitum) y una muestra de sangre de $5 \mathrm{ml}$ en ayuno. Además, se cuantificaron peso y talla y se les tomó una muestra independiente de orina (en ayuno) para efectuar minicultivo y medición de $\mathrm{pH}$ urinario.

La superficie corporal se determinó con la fórmula de Dubois-Dubois ${ }^{11}$. Se consideró una muestra de orina bien recogida cuando la excreción de creatinina se encontraba $< \pm 1$ desviación estándar (DE) con respecto al valor de referencia, de acuerdo con la edad, 
sexo y talla del sujeto ${ }^{12}$. El estado nutricional de los pacientes se estableció según el índice de masa corporal (IMC) en $\mathrm{kg} / \mathrm{m}^{2}$; los pacientes se clasificaron con desnutrición (<18.5), peso normal (18.5-24.9), sobrepeso $(25-29.9)$ y obesidad $(\geq 30)^{13}$.

Las determinaciones analíticas se realizaron mediante técnica espectrofotométrica, con un autoanalizador HumaStar 80 de la firma Human Diagnostics, Magdeburgo, Alemania.

Se consideraron los siguientes valores de corte para el diagnóstico de los trastornos metabólicos: hipercalcemia (calcio plasmático total > $10.2 \mathrm{mg} / \mathrm{dl} \quad 0$ $2.55 \mathrm{mmol} / \mathrm{l}$ ), hipofosfatemia (fósforo plasmático $<2.5 \mathrm{mg} / \mathrm{dl} \circ 0.8 \mathrm{mmol} / \mathrm{l}$ ), hiperuricemia (ácido úrico plasmático $>7 \mathrm{mg} / \mathrm{dl}$ o $416 \mu \mathrm{mol} / \mathrm{l}$ en hombres y > $5.7 \mathrm{mg} / \mathrm{dl}$ o $340 \mu \mathrm{mol} / \mathrm{l}$ en mujeres), hipercalciuria (calcio urinario $>4.0 \mathrm{mg} \circ 0.10 \mathrm{mmol} / \mathrm{kg} / \mathrm{día}$ ), hiperuricosuria (ácido úrico urinario $>800 \mathrm{mg} 04.76 \mathrm{mmol} / \mathrm{día}$ en hombres y > $750 \mathrm{mg}$ o $4.46 \mathrm{mmol} /$ día en mujeres), hiperoxaluria (oxalato urinario $>45 \mathrm{mg} 00.5 \mathrm{mmol} / \mathrm{día}$ ), hipocitraturia (citrato urinario $<350 \mathrm{mg} 01.82 \mathrm{mmol} /$ día), hipomagnesuria (magnesio urinario $<60 \mathrm{mg} 0$ $2.88 \mathrm{mmol} / \mathrm{d}$ áa), excreción de sodio aumentada (sodio urinario $>3 \mathrm{meq} / \mathrm{kg} / \mathrm{día}$ ), flujo urinario bajo (volumen urinario $<20 \mathrm{ml} / \mathrm{kg} / \mathrm{día}$ ) y $\mathrm{pH}$ urinario ácido (en ayuno) $<5.3^{14}$. La función renal se midió con depuración de creatinina en $\mathrm{ml} / \mathrm{min} / 1.73 \mathrm{~m}^{2} \mathrm{SC}$. La cistinuria y la ITU se diagnosticaron de forma cualitativa.

El estudio recibió aprobación del consejo científico y el comité de ética de la investigación del Instituto de Nefrología "Dr. Abelardo Buch López". Todos los pacientes concedieron su consentimiento informado para participar en la investigación. Se garantizó la confidencialidad de los datos recogidos durante el estudio.

\section{Estadística}

La totalidad de la información se procesó de forma automatizada. Se empleó el paquete estadístico Statistical Package for Social Science (SPSS) versión 22.0 para Windows (Chicago, Illinois, USA). Para las variables cualitativas se utilizó la técnica estadística de análisis de distribución de frecuencias; en cada una de las categorías de las variables estudiadas se calcularon las frecuencias absolutas y relativas (porcentajes). Para las variables cuantitativas se determinaron la media y la DE.

Para identificar las posibles relaciones entre las características de los pacientes y los trastornos metabólicos se usó la prueba de independencia, sin corrección por continuidad en el caso de la edad y con corrección
Tabla 1. Características demográficas de los pacientes estudiados

\begin{tabular}{|l|c|c|c|}
\hline Variable & Característica & No. & $\%$ \\
\hline Edad (años) & $60-64$ & 182 & 43.3 \\
\hline & $65-69$ & 127 & 30.2 \\
\hline $70-74$ & 81 & 19.3 \\
\hline Sexo & $75-79$ & 25 & 6.0 \\
\hline & Masculino & 223 & 53.1 \\
\hline
\end{tabular}

en el sexo y la valoración nutricional. En esta última variable fue necesario colapsar las tablas originales a tablas de dos filas y dos columnas, por encontrarse en todas las frecuencias esperadas menores del $5 \%$ en cantidades superiores a las requeridas para aplicar la prueba sin corrección. Para todas las pruebas de hipótesis efectuadas se fijó un nivel de significación de $\alpha=0.05$.

\section{Resultados}

La edad media de los 420 pacientes estudiados fue de 66.4 años, con una DE de 5.01 años; los de menor edad tenían 60 años y el de mayor edad tenía 82 . La mayoría de los pacientes (73.5\%) era menor de 70 años de edad (Tabla 1). Del total de pacientes, $223(53.1 \%)$ eran del sexo masculino, con una relación hombre/mujer de 1.13/1 (Tabla 1). La función renal promedio, medida por depuración de creatinina, fue de $65.4 \mathrm{ml} / \mathrm{min} / 1.73 \mathrm{~m}^{2} \mathrm{SC}$ (DE- $15.5 \mathrm{ml} / \mathrm{min} / 1.73 \mathrm{~m}^{2} \mathrm{SC}$ ). Tres pacientes $(0.7 \%)$ tenían un IMC que los llevó a clasificarse como desnutridos, 127 (30.2\%) eran de peso normal, 172 (41\%) mostraban sobrepeso y $118(28.1 \%)$ eran obesos.

Los valores promedio de las cuantificaciones plasmáticas y urinarias por grupos de edad pueden observarse en la Tabla 2. Las mayores diferencias se hallaron en el volumen urinario y las excreciones urinarias de citrato y ácido úrico.

Del total de pacientes estudiados, 401 (95.5\%) tenían al menos un trastorno metabólico. Los trastornos metabólicos más comunes en los pacientes estudiados fueron, en orden de frecuencia (Tabla 3), hipomagnesuria $(43.9 \%)$, excreción de sodio aumentada (39.9\%) y pH urinario ácido (32.4\%). 
Nefro Latinoam. 2021;18

Tabla 2. Valores promedio de las mediciones plasmáticas y urinarias por grupos de edad

\begin{tabular}{|c|c|c|c|c|}
\hline Variable & $\begin{array}{l}\text { 60-66 } \\
\text { años }\end{array}$ & $\begin{array}{l}\text { 67-73 } \\
\text { años }\end{array}$ & $\begin{array}{l}\text { 74-82 } \\
\text { años }\end{array}$ & Total \\
\hline Calcio sérico (mg/dl) & 9.1 & 9.1 & 9.3 & 9.1 \\
\hline Fósforo sérico (mg/dl) & 3.0 & 3.1 & 3.1 & 3.0 \\
\hline Ácido úrico sérico (mg/dl) & 5.4 & 5.2 & 5.7 & 5.4 \\
\hline Volumen urinario (ml) & $2,082.9$ & $1,953.6$ & $2,048.1$ & $2,036.6$ \\
\hline pH urinario & 5.73 & 5.79 & 5.72 & 5.75 \\
\hline $\begin{array}{l}\text { Excreción urinaria de } \\
\text { calcio }(\mathrm{mg})\end{array}$ & 155.8 & 132.4 & 144.7 & 146.9 \\
\hline $\begin{array}{l}\text { Excreción urinaria de } \\
\text { sodio (meq) }\end{array}$ & 212.2 & 193.6 & 203.1 & 205.1 \\
\hline $\begin{array}{l}\text { Excreción urinaria de } \\
\text { magnesio }(\mathrm{mg})\end{array}$ & 69.3 & 68.2 & 67.3 & 68.7 \\
\hline $\begin{array}{l}\text { Excreción urinaria de } \\
\text { citrato }(\mathrm{mg})\end{array}$ & 479.7 & 429.8 & 442.3 & 459.4 \\
\hline $\begin{array}{l}\text { Excreción urinaria de } \\
\text { oxalato }(\mathrm{mg})\end{array}$ & 18.4 & 17.4 & 16.0 & 17.8 \\
\hline $\begin{array}{l}\text { Excreción urinaria de } \\
\text { ácido úrico (mg) }\end{array}$ & 405.6 & 357.7 & 388.3 & 387.9 \\
\hline
\end{tabular}

Tabla 3. Trastornos metabólicos de los pacientes estudiados

\begin{tabular}{|l|c|c|}
\hline Trastornos & No./Total de muestras & $\%$ \\
\hline Hipomagnesuria & $183 / 417$ & 43.9 \\
\hline Excreción de sodio aumentada & $161 / 404$ & 39.9 \\
\hline pH urinario ácido & $136 / 420$ & 32.4 \\
\hline Volumen urinario bajo & $108 / 420$ & 25.7 \\
\hline Hipocitraturia & $98 / 418$ & 23.3 \\
\hline Infección del tracto urinario & $84 / 420$ & 20.0 \\
\hline Hiperuricemia & $70 / 420$ & 16.7 \\
\hline Hipofosfatemia & $52 / 420$ & 12.4 \\
\hline Hipercalciuria & $34 / 420$ & 8.1 \\
\hline Hiperuricosuria & $10 / 420$ & 2.4 \\
\hline Hipercalcemia & $8 / 420$ & 1.9 \\
\hline Hiperoxaluria & $5 / 418$ & 1.2 \\
\hline Cistinuria & $4 / 420$ & 1.0 \\
\hline
\end{tabular}

El análisis de los trastornos metabólicos renales más comunes de acuerdo con la edad de los pacientes (Tabla 4) muestra que la hiperuricemia fue la alteración
Tabla 4. Pacientes de acuerdo con los trastornos metabólicos más frecuentes encontrados y edad

\begin{tabular}{|l|c|c|c|c|c|c|}
\hline & \multicolumn{2}{|c|}{$60-66$ años } & \multicolumn{2}{|c|}{$\mathbf{6 7 - 7 3}$ años } & \multicolumn{2}{|c|}{$74-82$ años } \\
\cline { 2 - 7 } Trastornos & No. & $\%$ & No. & $\%$ & No. & $\%$ \\
\hline Hipomagnesuria & 101 & 41.7 & 59 & 44.0 & 22 & 53.7 \\
\hline $\begin{array}{l}\text { Excreción de sodio } \\
\text { aumentada }\end{array}$ & 93 & 40.8 & 52 & 38.8 & 16 & 38.1 \\
\hline pH urinario ácido & 75 & 31.5 & 48 & 34.5 & 13 & 30.2 \\
\hline $\begin{array}{l}\text { Volumen urinario bajo } \\
\text { Hipocitraturia }\end{array}$ & 51 & 25.6 & 36 & 25.9 & 11 & 25.6 \\
\hline $\begin{array}{l}\text { Infección del tracto } \\
\text { urinario }\end{array}$ & 42 & 17.6 & 29 & 20.9 & 13 & 30.2 \\
\hline Hiperuricemia* & 34 & 14.3 & 22 & 15.8 & 14 & 32.6 \\
\hline Hipofosfatemia & 32 & 13.4 & 15 & 10.8 & 5 & 11.6 \\
\hline Hipercalciuria & 18 & 7.6 & 11 & 7.9 & 5 & 11.6 \\
\hline * & & & & & 15 & 34.9 \\
\hline 0.05 & & & & & \\
\hline
\end{tabular}

que experimentó un mayor incremento de su presentación con el paso de la edad, con frecuencia de 14.3\% en los sujetos de 60 a 66 años, y de $32.6 \%$ en los mayores de 74 años, una diferencia estadísticamente significativa.

Al evaluar la frecuencia de los trastornos metabólicos de acuerdo con el sexo de los pacientes (Tabla 5) se advirtió que la hipomagnesuria, la hipocitraturia y la ITU fueron más comunes en las mujeres que en los hombres, con frecuencias respectivas en hombres y mujeres de $34.2 \%$ contra $54.9 \%, 16.6 \%$ contra $31.3 \%$ y 11.2 contra $29.9 \%$. Estas diferencias resultaron estadísticamente significativas. Por su parte, el pH urinario ácido y la hiperuricemia fueron más comunes en los individuos del sexo masculino, con frecuencias respectivas en hombres y mujeres de $40.4 \%$ contra $23.4 \%$ y $21.1 \%$ contra $11.7 \%$, también con significación estadística. El resto de los trastornos no mostraron diferencias muy notorias entre los sexos.

El estado nutricional de los pacientes se vinculó con la frecuencia de presentación de algunos de los trastornos metabólicos (Tabla 6). En consecuencia, la excreción de sodio aumentada y la hipercalciuria fueron significativamente más frecuentes en los pacientes clasificados como desnutridos y con peso normal (51.2\% y $15.4 \%$ ) respecto de aquéllos con sobrepeso y obesidad (34.8\% y 4.8\%); en cambio, el volumen urinario disminuido fue más común entre los individuos con sobrepeso y obesidad (31.7\%) en relación con los desnutridos y de 
Tabla 5. Pacientes de acuerdo con los trastornos metabólicos más frecuentes encontrados y sexo

\begin{tabular}{|l|c|c|c|c|}
\hline \multirow{2}{*}{ Trastornos } & \multicolumn{2}{|c|}{ Masculino } & \multicolumn{2}{|c|}{ Femenino } \\
\cline { 2 - 5 } & No. & $\%$ & No. & $\%$ \\
\hline Hipomagnesuria** & 76 & 34.2 & 107 & 54.9 \\
\hline Excreción de sodio aumentada & 95 & 44.4 & 66 & 34.7 \\
\hline pH urinario ácido** & 90 & 40.4 & 46 & 23.4 \\
\hline Volumen urinario bajo & 61 & 27.4 & 47 & 23.9 \\
\hline Hipocitraturia** & 37 & 16.6 & 61 & 31.3 \\
\hline $\begin{array}{l}\text { Infección del tracto urinario** } \\
\text { Hiperuricemia* }\end{array}$ & 25 & 11.2 & 59 & 29.9 \\
\hline $\begin{array}{l}\text { Hipofosfatemia } \\
\text { Hipercalciuria }\end{array}$ & 47 & 21.1 & 23 & 11.7 \\
\hline $\begin{array}{l}\text { * } p<0.05 \\
\text { ** } p<0.01\end{array}$ & 34 & 15.2 & 18 & 9.1 \\
\hline
\end{tabular}

peso normal (12.3\%). No se identificaron otras diferencias estadísticamente significativas entre las frecuencias de los trastornos de acuerdo con el IMC.

\section{Discusión}

Los pacientes mayores de 60 años son un grupo poblacional que experimenta un notorio crecimiento a escala planetaria y tiene más presentaciones atípicas y peor evolución de las urolitiasis que los individuos más jóvenes ${ }^{5,15,16}$. Por consiguiente, las acciones preventivas para las urolitiasis, tanto primarias como secundarias, cobran particular importancia en estos individuos ${ }^{14}$. Sin embargo, los estudios relativos a los trastornos metabólicos renales que producen las urolitiasis en estas edades son muy escasos, lo que impide hacer intervenciones específicas para este grupo poblacional ${ }^{10}$. Además, limita contrastar los resultados de este estudio.

El mayor número de pacientes menores de 70 años era un resultado esperado, dado que se corresponde con los estudios epidemiológicos nacionales e internacionales $^{17-19}$. De modo semejante, el ligero predominio masculino identificado se corresponde con los hallazgos de grandes series internacionales, aunque en los últimos años las diferencias de prevalencia de urolitiasis entre sexos han disminuido, incluidos los pacientes mayores de 60 años ${ }^{17,20,21}$.

La elevada frecuencia de trastornos metabólicos identificada en esta serie se corresponde con la informada de Amaro, et al., que fue del $96.8 \%$ en 735 pacientes brasileños mayores de 12 años, lo que subraya la importancia del estudio en los adultos mayores $^{22}$. Como grupo, las frecuencias identificadas de los diferentes trastornos metabólicos son muy diferentes a las encontradas en la población cubana adulta una década atrás, lo que también destaca la necesidad de intervenciones particulares en estas edades ${ }^{23}$. Por lo tanto, resulta muy llamativa la frecuencia de hipercalciuria, que es muy inferior a la identificada en el estudio de adultos cubanos en que superó el $45 \%$, en tanto que la hipocitraturia y el volumen urinario bajo fueron mucho más frecuentes que en el estudio ya citado ${ }^{23}$. Es necesario investigar si la menor frecuencia de hipercalciuria en pacientes mayores de 60 años respecto de individuos más jóvenes se limita a la población cubana 0 es un fenómeno más general.

Al comparar los resultados de este trabajo con el de Freitas, et al. desarrollado en hombres mayores de 60 años brasileños (51 pacientes, 26 casos y 25 controles), Ilaman la atención las elevadas frecuencias de hipernatruria, hipocitraturia y $\mathrm{pH}$ urinario ácido identificadas en ambos estudios, aunque superiores en el trabajo brasileño ${ }^{10}$. Debe considerarse que en el estudio brasileño no se hicieron mediciones de magnesio urinario, de tal manera que no puede contrastarse la alta frecuencia de hipomagnesuria hallada en este trabajo.

El hallazgo más llamativo de esta investigación es la frecuencia elevada de hipomagnesuria que coincide con otros estudios de adultos que han evaluado esta variable (con puntos de corte semejantes para la definición del trastorno). Hussein, et al. identificaron una frecuencia de hipomagnesuria del 59.3\% en 96 pacientes adultos malasios y Amaro, et al. del $50.1 \%$ en adultos brasileños; empero, otros trabajos como el de Spivacow, et al. en Argentina, han identificado una frecuencia muy inferior $(8.86 \%$ entre 3,014 adultos) $)^{22,24,25}$. El magnesio no se considera un inhibidor tan potente de la litogénesis como el citrato, pero los análisis químicos han revelado una relación entre la hipomagnesuria y las litiasis de oxalato de calcio dihidratadas, que son de las más frecuentes en la población cubana adulta ${ }^{26-28}$. Se precisan otras investigaciones en adultos mayores litiásicos que exploren la magnesuria.

La elevada frecuencia de excreción de sodio aumentada identificada guarda relación con lo hallado en individuos litiásicos adultos cubanos, y tiene particular interés porque ésta se suele acompañar de otras alteraciones metabólicas que producen litogénesis, como 
la hipocitraturia y la hipercalciuria, además de influir en el volumen urinario ${ }^{23,29,30}$. En los pacientes mayores, el aumento de la ingestión de sal parece depender de modificaciones del gusto ${ }^{31}$.

El pH urinario ácido si bien no constituye propiamente un trastorno metabólico porque el pH urinario está condicionado por la carga ácida de la dieta, casi siempre se toma en consideración en estudios de esta naturaleza dado que el pH urinario ácido propicia la litogénesis (especialmente la de ácido úrico) ${ }^{32}$. La alta frecuencia de orinas ácidas no es de extrañar, si se consideran los resultados de Menezes, et al., que estudiaron a 7,891 pacientes litiásicos de Estados Unidos, 1332 de los cuales eran mayores de 64 años, e identificaron una caída sostenida del pH urinario desde la juventud, que parece explicarse por varios factores, entre ellos la caída de la tasa de filtración glomerular, el incremento del IMC, la disminución de la amoniuria y la acidez titulable, las modificaciones dietéticas, los cambios en el manejo gastrointestinal de los aniones y el incremento de la excreción urinaria de potasio ${ }^{33}$.

Resultó muy notorio el incremento de la frecuencia de hiperuricemia con la edad, algo que se corresponde con los resultados del estudio de Zitt, et al., que en una gran cohorte de personas (146,873 participantes) de población general en Austria identificó un aumento sostenido de los valores de ácido úrico con la edad, incluidos los sujetos mayores de 60 años $^{34}$.

Las diferencias en la frecuencia de los trastornos por sexo revisten mucho interés porque son un elemento muy importante a considerar en la personalización de la prevención de las urolitiasis. El predominio de la hipomagnesuria en las mujeres no se ha descrito con anterioridad. Por su parte, la mayor frecuencia de hipocitraturia en el sexo femenino resulta muy interesante, dado que Parks y Coe reconocieron una mayor excreción de citrato en las mujeres, pero Curhan, et al. no confirmaron estos hallazgos en un grupo numeroso de litiásicos que tomaron parte en tres estudios poblaciones de cohorte desarrollados en Estados Unidos $^{35,36}$. Se requieren entonces otros estudios en mujeres posmenopáusicas que confirmen estos resultados.

La ITU, como era de esperar a partir de los estudios en población adulta y en pacientes litiásicos, fue más común en las mujeres, lo que depende de las características anatómicas de la uretra femenina, entre otros factores $^{37,38}$. No debe perderse de vista que la litiasis constituye un factor de riesgo para el desarrollo de ITU y una complicación de éstas ${ }^{14,32}$.
Se podía prever el predominio de la hiperuricemia en hombres, dado el comportamiento de los valores de ácido úrico en la población general y los pacientes adultos litiásicos ${ }^{34,39}$, aunque no debe soslayarse que luego de los 50 años de edad las mujeres suelen experimentar una elevación de las concentraciones séricas de ácido úrico, tal vez por la pérdida del efecto uricosúrico de los estrógenos ${ }^{34}$. Asimismo, la mayor frecuencia de las orinas ácidas en los individuos del sexo masculino se corresponde con los hallazgos en la población litiásica adulta y parecen determinadas por diferencias en la generación ácida ${ }^{33}$.

La evaluación de los trastornos metabólicos en el contexto del estado nutricional de los sujetos es un elemento de mucho peso en el paciente mayor de 60 años, ya que hoy en día la urolitiasis se considera una enfermedad sistémica relacionada con la obesidad, la ganancia de peso en la adultez y los estados de resistencia a la insulina ${ }^{9,14}$. Sin embargo, los trastornos vinculados con el estado nutricional fueron aquellos cuyo valor de corte para la definición del trastorno se hallaba en función del peso corporal, es decir, excreción de sodio aumentada, hipercalciuria y volumen urinario bajo. Por consiguiente, en los dos primeros su mayor frecuencia en los pacientes desnutridos y con peso normal pudo determinarse por el menor peso corporal, puesto que con un mismo grado de excreción que un individuo de la otra categoría (con sobrepeso y obesidad) se clasificarían como afectados por el trastorno; lo mismo ocurre, pero en sentido opuesto, para el volumen urinario bajo. Es cierto que, al margen de lo antes señalado, podría haber una base biológica que explicara, al menos en parte, las diferencias, pero para emitir tales afirmaciones se necesitan valores de corte que no estén desarrollados en función del peso.

Este trabajo tiene como limitaciones la realización de un solo estudio metabólico por paciente, cuando parece recomendable el estudio de un número mayor de muestras ${ }^{40}$, la utilización de valores de corte para la definición de los trastornos, desarrollados a partir de adultos jóvenes que no toman en consideración los efectos del envejecimiento, la falta de estudios de acidificación urinaria y las cuantificaciones de hormona paratiroidea que habrían permitido mayores precisiones diagnósticas, así como la falta de consignación de los antecedentes de enfermedades, como diabetes mellitus, hipertensión arterial, cardiopatía isquémica o síndrome metabólico, que se han relacionado con el desarrollo de urolitiasis ${ }^{14}$. No obstante, este estudio se ha desarrollado con un número considerable de individuos provenientes de todo el país (constituye la serie 
Tabla 6. Pacientes de acuerdo con los trastornos metabólicos más frecuentes encontrados y estado nutricional

\begin{tabular}{|c|c|c|c|c|}
\hline \multirow[t]{2}{*}{ Trastornos } & \multicolumn{2}{|c|}{$\begin{array}{c}\text { Desnutridos-peso } \\
\text { normal } \\
\left(\text { IMC }<25 \mathrm{~kg} / \mathrm{m}^{2}\right)\end{array}$} & \multicolumn{2}{|c|}{$\begin{array}{c}\text { Sobrepeso- } \\
\text { obesidad } \\
\left(\text { IMC } \geq 25 \mathrm{~kg} / \mathrm{m}^{2}\right)\end{array}$} \\
\hline & No. & $\%$ & No. & $\%$ \\
\hline Hipomagnesuria & 58 & 45.3 & 125 & 43.2 \\
\hline $\begin{array}{l}\text { Excreción de sodio } \\
\text { aumentada* }\end{array}$ & 64 & 51.2 & 97 & 34.8 \\
\hline pH urinario ácido & 40 & 30.8 & 96 & 33.1 \\
\hline Volumen urinario bajo* & 16 & 12.3 & 92 & 31.7 \\
\hline Hipocitraturia & 32 & 24.6 & 66 & 22.9 \\
\hline $\begin{array}{l}\text { Infección del tracto } \\
\text { urinario }\end{array}$ & 24 & 18.5 & 60 & 20.7 \\
\hline Hiperuricemia & 16 & 12.3 & 54 & 18.6 \\
\hline Hipofosfatemia & 16 & 12.3 & 36 & 12.4 \\
\hline Hipercalciuria* & 20 & 15.4 & 14 & 4.8 \\
\hline
\end{tabular}

más grande de su tipo), con un mismo protocolo, y constituye la mayor evidencia disponible sobre los trastornos metabólicos renales que predisponen a las urolitiasis en la población mayor de 60 años de edad, de tal modo que puede servir como referencia para el diseño de medidas de prevención primaria y secundaria de las urolitiasis dirigidas a estos pacientes.

\section{Conclusiones}

Los trastornos metabólicos renales son muy comunes en pacientes litiásicos cubanos mayores de 60 años y los más frecuentes son la hipomagnesuria, la excreción urinaria de sodio aumentada y el pH urinario ácido. La hiperuricemia es más común en pacientes de mayor edad. La hipomagnesuria, la hipocitraturia y la ITU son más frecuentes en las mujeres, mientras el pH urinario ácido y la hiperuricemia los son en los hombres. La excreción urinaria de sodio aumentada y la hipercalciuria son más comunes en desnutridos y con peso normal, en tanto que el volumen urinario disminuido lo es en individuos con sobrepeso y obesos.

\section{Financiamiento}

Los autores no tienen fuentes de financiamiento que declarar.

\section{Agradecimientos}

Los autores laboran en el Instituto de Nefrología "Dr. Abelardo Buch López", La Habana, Cuba.

\section{Conflicto de intereses}

Los autores declaran que no existe ningún conflicto de intereses (económico, profesional o personal) real o potencial que pueda resultar en un sesgo en la publicación de este trabajo.

\section{Responsabilidades éticas}

Protección de personas y animales. Los autores declaran que para esta investigación no se han realizado experimentos en seres humanos ni en animales.

Confidencialidad de los datos. Los autores declaran que han seguido los protocolos de su centro de trabajo sobre la publicación de datos de pacientes.

Derecho a la privacidad y consentimiento informado. Los autores han obtenido el consentimiento informado de los pacientes o sujetos referidos en el artículo. Este documento obra en poder del autor de correspondencia.

\section{Bibliografía}

1. Raheem OA, Khandwala YS, Sur RL, Ghani KR, Denstedt JD. Burden of urolithiasis: trends in prevalence, treatments, and costs. Eur Urol Focus. 2017;3(1):18-26.

2. Ziemba JB, Matlaga BR. Epidemiology and economics of nephrolithiasis. Investig Clin Urol. 2017;58(5):299-306.

3. Ferraro PM, Curhan GC, D'Addessi A, Gambaro G. Risk of recurrence of idiopathic calcium kidney stones: analysis of data from the literature. J Nephrol. 2017;30(2):227-33.

4. Situación de la enfermedad renal crónica en Cuba. 2015. Anuario Cuba Nefro-Red 2014. [Internet] Accessed January, 2020. http://www.sld.cu/ sitios/nefrologia

5. World report on ageing and health. Geneva: World Health Organization (WHO);2015 (http://www.who.int/ageing/publications/world-report-2015, accessed 6 July 2020).

6. Biggs A, Bloom D, Burtless G, Fujiwara M, Hayashi K, Kanzler L, et al. A slow burning fuse: a special report on ageing populations. The Economist. 2009(27):1-15.

7. Oficina Nacional de Estadística e Información (ONEI). República de Cuba. Anuario Estadístico de Cuba 2018. Edición 2019. [Internet] Accessed in April, 2019: Available from. www.one.cu/aec2019.htm

8. Bonet-Gorbea M, Varona-Pérez P, Chang-La Rosa M, García-Rocha RG, Suárez-Medina R, Arcia-Montes de Oca N, et al. III Encuesta nacional de factores de riesgo y actividades preventivas de enfermedades no transmisibles. Cuba 2010-2011. 1ra ed. La Habana, Cuba: Editorial Ciencias Médicas; 2015. Capítulo 9. Evaluación antropométrica; p.110-40.

9. Carbone A, Al Salhi Y, Tasca A, Palleschi G, Fuschi A, De Nunzio C, et al. Obesity and kidney stone disease: a systematic review. Minerva Urol Nefrol. 2018 Aug;70(4):393-400.

10. Freitas-Junior CH, Mazzucchi E, Danilovic A, Brito AH, Srougi M. Metabolic assessment of elderly men with urolithiasis. Clinics (Sao Paulo). 2012;67(5):457-61.

11. Dubois D, Dubois EF. A formula to estimate the approximate surface area if height and weight be known. Arch Intern Med. 1916;17:862.

12. Badell-Moore A, Bacallao-Méndez RA. Sobre el establecimiento de valores de referencia de la excreción de creatinina para la población cubana. Rev Cubana Aliment Nutr. 2015;25(1 Supl): S28-S60.

13. Garrow JS, Webster J. Quetelet's index $\left(\mathrm{W} / \mathrm{H}^{2}\right)$ as a measure of fatness. Int J Obes. 1985;9:147-53. 
14. Sakhaee K, Moe OW. Urolithiasis. En: Skorecki K, Chertow GM Marsden PA, Taal MW, Yu ASL, editors. The kidney. 10 ed. Philadelphia: Elsevier Health Sciences; 2016:1322-67.

15. Dursun M, Ozbek E, Otunctemur A, Sahin S, Cakir SS. Clinical presentation of urolithiasis in older and younger population. Arch Ital Urol Androl. 2014;86(4):249-52.

16. Arampatzis $S$, Lindner G, Irmak F, Funk GC, Zimmermann $H$ Exadaktylos AK. Geriatric urolithiasis in the emergency department: risk factors for hospitalisation and emergency management patterns of acute urolithiasis. BMC Nephrol. 2012 Sep 24;13:117. doi: 10.1186/14712369-13-117.

17. Stamatelou KK, Francis ME, Jones CA, Nyberg LM, Curhan GC. Time trends in reported prevalence of kidney stones in the United States: 1976-1994. Kidney Int. 2003:63(5):1817-23.

18. Moudi E, Hosseini SR, Bijani A. Nephrolithiasis in elderly population; effect of demographic characteristics. J Nephropathol. 2017 Mar;6(2):63-8.

19. Bacallao-Méndez RA, Victores-Aguiar I, Mañalich-Comas R, Gutiérrez-García F, Llerena-Ferrer B, Almaguer-López MM. Caracterización clínico epidemiológica de la litiasis urinaria en un área rural de Artemisa. Rev Cubana Invest Bioméd. 2016;35(4):300-10.

20. Scales CD, Smith AC, Hanley JM, Saigal CS; Urologic Diseases in America Project. Prevalence of kidney stones in the United States. Eur Urol. 2012 Jul;62(1):160-5

21. Romero V, Akpinar $\mathrm{H}$, Assimos DG. Kidney stones: a global picture of prevalence, incidence, and associated risk factors. Rev Urol. 2010 Spring;12(2-3):e86-96.

22. Amaro CR, Goldberg J, Damasio PC, Leitão VA, Turney B, Padovani CR Amaro JL. An update on metabolic assessment in patients with urinary lithiasis. World J Urol. 2015 Jan;33(1):125-9.

23. Bacallao-Méndez RA, Madrid-Mancia C, Mañalich-Comas R, Gutiérrez-García F, Badell-Moore A. Trastornos metabólicos renales en pacientes cubanos adultos con litiasis urinarias. Rev Cubana Med. 2014;53(4):456-67.

24. Hussein NS, Sadiq SM, Kamaliah MD, Norakmal AW, Gohar MN. Twenty-four-hour urine constituents in stone formers: a study from the northeast part of Peninsular Malaysia. Saudi J Kidney Dis Transpl. 2013 May;24(3):630-7.

25. Spivacow FR, del Valle EE, Negri AL, Fradinger E, Abib A, Rey P. Biochemical diagnosis in 3040 kidney stone formers in Argentina. Urolithiasis. 2015 Aug;43(4):323-30.
26. Massey L. Magnesium therapy for nephrolithiasis. Magnes Res. 2005 Jun;18(2):123-6

27. Bibilash BS, Vijay A, Fazil Marickar YM. Stone composition and metabolic status. Urol Res. 2010 Jun;38(3):211-3.

28. Bacallao-Méndez RA, Mañalich-Comas R, Gutiérrez-García F, BadeII-Moore A. Composición de las urolitiasis en pacientes cubanos por sexo. Rev Cubana Invest Bioméd. 2015;34(4):328-36.

29. Ticinesi A, Nouvenne A, Maalouf NM, Borghi L, Meschi T. Salt and nephrolithiasis. Nephrol Dial Transplant. 2016 Jan;31(1):39-45.

30. Bacallao-Méndez RA, Caldevilla-Rodríguez Y, Mañalich-Comas R, Gutiérrez-García F, Badell-Moore A, Llerena-Ferrer B. Efecto de la ingestión de sal en la excreción renal de agua en pacientes urolitiásicos. Rev Cubana Invest Bioméd. 2016;35(4):323-30.

31. Sergi G, Bano G, Pizzato S, Veronese N, Manzato E. Taste loss in the elderly: possible implications for dietary habits. Crit Rev Food Sci Nutr. 2017:57(17):3684-89.

32. Maalouf N. Approach to the adult kidney stone former. Clin Rev Bone Miner Metab. 2012;10(1):38-49.

33. Menezes CJ, Worcester EM, Coe FL, Asplin J, Bergsland KJ, Ko B. Mechanisms for falling urine $\mathrm{pH}$ with age in stone formers. Am J Physiol Renal Physiol. 2019 Jul 1;317(7):F65-F72.

34. Zitt E, Fischer A, Lhotta K, Concin H, Nagel G. Sex- and age-specific variations, temporal trends and metabolic determinants of serum uric acid concentrations in a large population-based Austrian cohort. Sci Rep. 2020 May 5;10(1):7578. doi: 10.1038/s41598-020-64587-z

35. Parks JH, Coe FL. A urinary calcium-citrate index for the evaluation of nephrolithiasis. Kidney Int. 1986;30:85-90.

36. Curhan GC, Willett WC, Speizer FE, Stampfer MJ. Twenty-four-hour urine chemistries and the risk of kidney stones among women and men. Kidney Int. 2001;59:2290-8.

37. Alpay $\mathrm{Y}$, Aykin N, Korkmaz P, Gulduren HM, Caglan FC. Urinary tract infections in the geriatric patients. Pak J Med Sci. 2018;34(1):67-72.

38. Jackson SL, Boyko EJ, Scholes D, Abraham L, Gupta K, Fihn SD. Predictors of urinary tract infection after menopause: a prospective study. Am J Med. 2004; 117(12):903-11.

39. Wiederkehr MR, Moe OW. Uric acid nephrolithiasis: a systemic metabolic disorder. Clin Rev Bone Miner Metab. 2011 Dec;9(3-4):207-17.

40. Parks JH, Goldfisher E, Asplin JR, Coe FL. A single 24-hour urine collection is inadequate for the medical evaluation of nephrolithiasis. J Urol. 2002;167:1607-12. 